\title{
A Morphological and Immunohistochemical Study of the Tumoral and Inflammatory Cells in Pancreatic Ductal Adenocarcinoma
}

\author{
Vlad Herlea $\mathbb{D}^{1,2,3}$ Elena Stoica Mustafa, ${ }^{1}$ Andreea Cristina Iorgescu, ${ }^{1}$ \\ Nicolae Catalin Pechianu, ${ }^{1,2}$ Dragos Cretoiu, ${ }^{4}$ Maria Sajin, ${ }^{4}$ Simona Olimpia Dima, ${ }^{3,5}$ \\ Catalin Vasilescu, ${ }^{4,5}$ Cezar Stroescu, ${ }^{5}$ Constantin Ungureanu, ${ }^{5}$ Traian Dumitrascu, ${ }^{5}$ \\ Vladislav Brasoveanu, ${ }^{2,5}$ Minerva Ghinescu, $^{2}$ and Irinel Popescu ${ }^{2,3,5}$ \\ ${ }^{1}$ Department of Pathology, Fundeni Clinical Institute, Bucharest 022328, Romania \\ 2 "TituMaiorescu" University, Faculty of Medicine, Bucharest 031593, Romania \\ ${ }^{3}$ Center of Excellence for Translational Medicine, Fundeni Clinical Institute, Bucharest, Romania \\ 4 "Carol Davila" University of Medicine and Pharmacy, Bucharest 020021, Romania \\ ${ }^{5}$ Center of General Surgery and Liver Transplantation, Fundeni Clinical Institute, Bucharest 022328, Romania
}

Correspondence should be addressed to Vlad Herlea; herlea2002@yahoo.com

Received 30 June 2020; Revised 25 August 2020; Accepted 15 September 2020; Published 29 September 2020

Academic Editor: Monica Neagu

Copyright ( 2020 Vlad Herlea et al. This is an open access article distributed under the Creative Commons Attribution License, which permits unrestricted use, distribution, and reproduction in any medium, provided the original work is properly cited.

\begin{abstract}
This study is aimed at investigating tumoral and inflammatory cells and the significance of the prognostic factors of pancreatic ductal adenocarcinoma (PDAC); it is also aimed at determining the role of immunohistochemistry in the diagnosis and prognosis of this neoplasm. Materials and Methods. 230 cases of pancreatic ductal adenocarcinoma were included in the study group; these cases were selected from the archives of the Department of Pathology of the Fundeni Clinical Institute over a tenyear period. Immunohistochemistry was performed using the following antibodies: MUC 1, CD 34, Factor VIII, CD 68, MMP-7, CEA, p21, p53, and Ki 67. Results. There were 133 male (57.8\%) and 97 female (42.2\%) patients included in this study, with ages between 20 and 81 years old (mean age: 58.2 years) and with tumors located in the pancreatic head $(n=196 ; 85.2 \%)$, pancreatic body $(n=12 ; 5.2 \%)$, and pancreatic tail $(n=20,8.7 \%)$, as well as panpancreatic tumors $(n=2 ; 0.9 \%)$. Patients presented with early stages (IA and IB), with low pathologic grade (G1), with small size tumors (less than 1-1.5 cm), with tumors located in the head of the pancreas, (p53: negative; p21: positive; and CD 68: positive in peritumoral tissue), with low nuclear index (Ki $67<10 \%$ ), without metastases at the time of surgery (had a better prognosis), and with a survival rate of about 7 months. Conclusions. Immunohistochemistry is useful for an accurate diagnosis, differential diagnosis, and establishment of additional factors that might have a prognostic importance. It is recommended to study peritumoral tissue from the quantitative and qualitative points of view to increase the number of prognostic factors. This study represents a multidisciplinary approach, and it is a result of teamwork; it presents histopathological methods of examination of this severe illness and describes only a part of the scientific effort to determine the main pathological mechanisms of this neoplasm.
\end{abstract}

\section{Introduction}

Pancreatic cancer is an aggressive neoplasm with a poor prognosis. It is the fifth leading cause of death in Europe [1] and the fourth most common cause of cancer-related deaths in the United States [2], mostly because it is often discovered at an advanced stage [2-5]. The response to oncological therapy is limited [6], surgery being the only effective method of treatment for resectable tumors. $[2,7]$

Most of these neoplasms are represented by pancreatic ductal adenocarcinomas (85\%) [1]. It is rarely diagnosed in young people, and most of the patients are over 60 years old [8]. It grows and spreads very quickly. 
Diabetes and chronic pancreatitis are two of the most important risk factors, but prognostic factors are still debatable and challenging [9-12].

This study is aimed at investigating tumoral and inflammatory cells and the significance of prognostic factors of pancreatic ductal adenocarcinoma (PDAC); it is also aimed at determining the role of immunohistochemistry in the diagnosis and prognosis of this neoplasm.

\section{Materials and Methods}

230 cases of pancreatic ductal adenocarcinoma were included in the study group; these cases were selected from the archives of the Department of Pathology of the Fundeni Clinical Institute over a 10 -year period. The selection was made from radical surgical resected specimens. Duodenal, common bile duct, and ampullary neoplasms were excluded.

All cases were staged according to international criteria $[13,14]$. From the surgical specimens, tumoral and nontumoral tissue, resection margins, and lymph nodes were carefully sampled. Peritumoral inflammatory infiltrate was studied using a pancreatic tissue sample, taken $1 \mathrm{~cm}$ from tumoral/peritumoral junction.

Tissue samples were fixed in buffered formalin (10\%) for $10-20$ hours and embedded in paraffin $\left(57-58^{\circ} \mathrm{C}\right)$. From every paraffin block, serial sections were cut into 3-4 $\mu \mathrm{m}$ slices and then routinely stained with hematoxylin and eosin for histopathological diagnosis.

2.1. Morphological Analysis. The studied cases were distributed into the following 7 age groups: 20-29 years old, 30-39 years old, 40-49 years old, 50-59 years old, 60-69 years old, $70-79 y e a r s$ old, and over 80 years old. Age distribution was correlated with gender, size, and site of tumor. Tumor size was assessed in $\mathrm{cm}$, and the cases were distributed into 5 groups: $0.1-1.0 \mathrm{~cm}, 1.1-2.0 \mathrm{~cm}, 2.1-3.0 \mathrm{~cm}, 3.1-5.0 \mathrm{~cm}$, and over $5 \mathrm{~cm}$. The tumor site was distributed according to anatomical regions in the head, body, tail and, in some cases where the entire organ was affected, panpancreatic.

Histological characteristics assessed for each case were tumor grades G1, G2, and G3 (according to the guideline criteria in use $[12,13])$; the presence or absence of lymph node metastases $(\mathrm{N} 0 / \mathrm{N} 1)$; the presence or absence of vascular invasion $(\mathrm{V}+/ \mathrm{V}-)$; the presence or absence of perineural invasion $(\mathrm{Pn}+/ \mathrm{Pn}-)$; and the presence or absence of endoluminal necrosis $(\mathrm{Ne}+/ \mathrm{Ne}-)$.

2.2. Immunohistochemistry. For immunohistochemistry, we used the biotin/streptavidin method [15].

The following antibodies, assessed on $2-3 \mu \mathrm{m}$ thick sections cut from paraffin-embedded tissue (paraffin blocks) and put on special slides (Super frost, commercially available), were used: MUC 1 (Polyconal Antibody, Thermo Fisher Scientific), Ki 67 (Clone SP-6, Lab Vision, Thermo Fisher Scientific), CD 34 (Clone QBEnd/10, Lab Vision, Thermo Fisher Scientific), Factor VIII (Clone F8/86, Lab Vision, Thermo Fisher Scientific), CD 68 (Clone KP1, Lab Vision, Thermo Fisher Scientific), MMP-7 (Clone ID2, Lab Vision, Thermo Fisher Scientific), CEA (Clone COL-1, Lab
Vision, Thermo Fisher Scientific), p21 (Clone CP74, Lab Vision, Thermo Fisher Scientific), and p53 (Clone DO-1, Lab Vision, Thermo Fisher Scientific). Evaluation of the immunohistochemical stains was independently performed by two pathologists.

To estimate the immunohistochemical results, the following scale was established:

(a) $0=$ negative staining $(0-5 \%$ of the cells are stained)

(b) $1+=$ weak positivity staining $(5-10 \%$ of the cells are stained)

(c) $2+=$ moderate positivity staining $(10-50 \%$ of the cells are stained)

(d) $3+=$ strong positivity staining (over $50 \%$ of the cells are stained)

The percentage of positive cells was established by analyzing 500-1000 cells, and the ratio between the numbers of positive cells versus the total number of analyzed cells was represented. Immunohistochemical analysis of tumor's blood supply was made by using antibodies for CD 34 or Factor VIII. In order to the count positive markers for microvascularization, using literature criteria [16], it was considered not necessary for a vascular lumen to be present. There were 3 areas quantified at a magnitude of 100 (10x eyepiece, $10 \mathrm{x}$ objective) that were identified with the highest microvascularization, and the vessels were quantified at a magnitude of 200 (10x eyepiece, $20 x$ objective).

2.3. Statistical Analysis. Univariate comparisons were performed using Pearson's chi-squared test and Student's $t$-test, as appropriate. All statistical tests were two sided, and a level of $p<0.05$ was used to indicate statistical significance. Statistical analysis was performed using the Stata MP Version 13.0 (College Station, TX).

\section{Results}

The gender distribution for the patients included in this study is as follows: 133 males $(57.8 \%)$ and 97 females $(42.2 \%)$.

Tumor site distribution is as follows: in the pancreatic head, $n=196(85.2 \%)$; in the pancreatic body, $n=12$ (5.2\%); in the pancreatic tail, $n=20(8.7 \%)$; and for panpancreatic tumors, $n=2(0.9 \%)$ (Figures 1 and 2).

Age distribution showed one $(0.43 \%)$ case in the $20-29$ year-old group, $12(5.22 \%)$ cases in the 30-39-year-old group, $37(16.08 \%)$ cases in the $40-49$-year-old group, 77 $(33.48 \%)$ cases in the 50-59-year-old group, $72(31.32 \%)$ cases in the 60-69-year-old group, $30(13.04 \%)$ cases in the $70-79$-year-old group, and one $(0.43 \%)$ case in the last group over 80 years old.

Tumor size distribution is as follows: 32 cases in the $0.1-$ $1.0 \mathrm{~cm}$ group, 50 cases in the $1.1-2.0 \mathrm{~cm}$ group, 70 cases in the $2.1-3.0 \mathrm{~cm}, 46$ cases in the $3.1-5.0 \mathrm{~cm}$, and 15 cases in the last group of over $5 \mathrm{~cm}$. In 17 cases, the size of the tumor could not be assessed (Figure 3). 


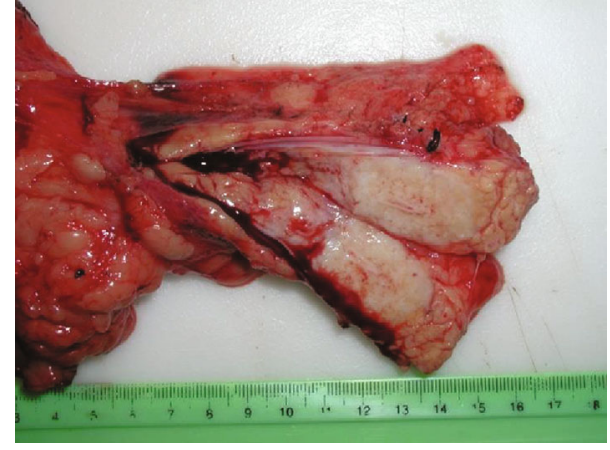

Figure 1: Surgically resected specimen: pancreatic tumor (gross appearance).

Tumor site distribution

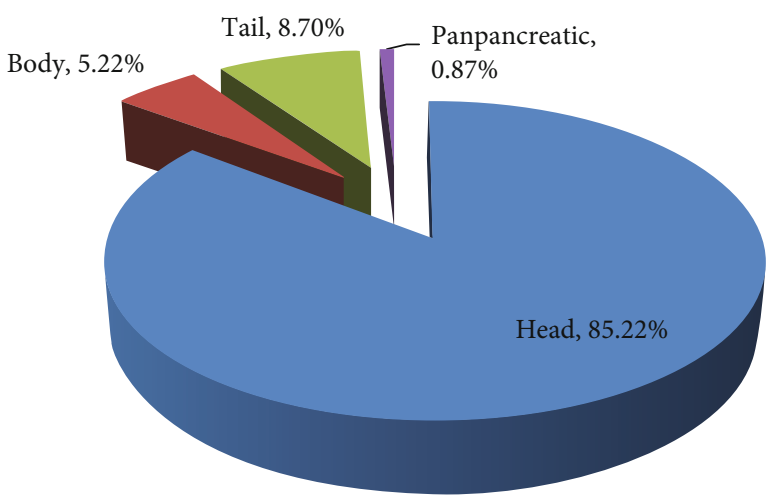

FIGURE 2: Tumor site distribution.

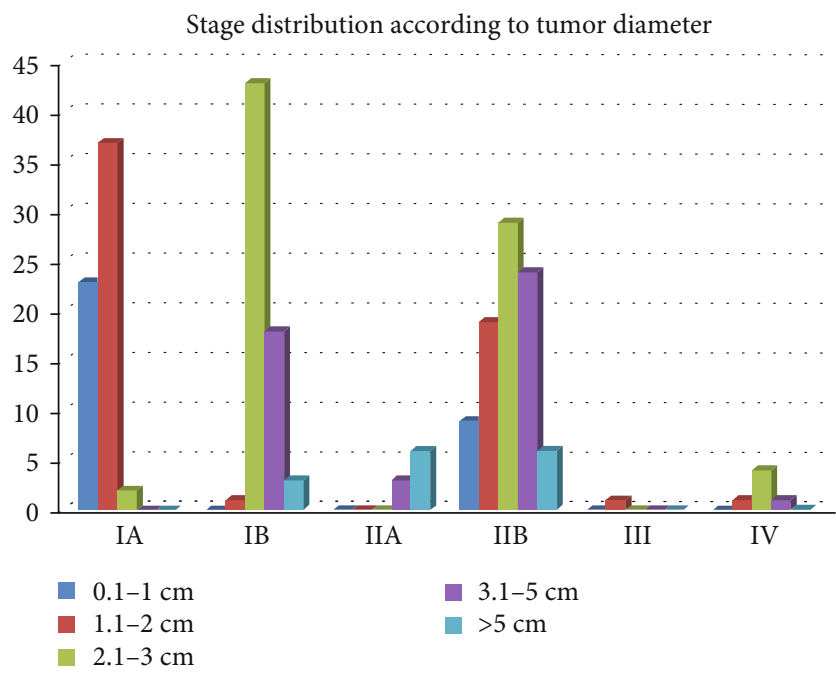

FIgURE 3: Stage distribution according to tumor diameter.

According to the TNM staging system $[1,12,13]$, tumor stage distribution was as follows: $38.3 \%$ in the pT1 group, $55.2 \%$ in the pT2 group, $6.1 \%$ in the pT3 group, and $0.4 \%$ in the pT4 group; $27.0 \%$ of the cases were stage IA, $28.3 \%$ were stage IB, $3.9 \%$ were stage IIA, $37.8 \%$ were stage IIB, $0.4 \%$ were stage III, and $2.6 \%$ were stage IV.

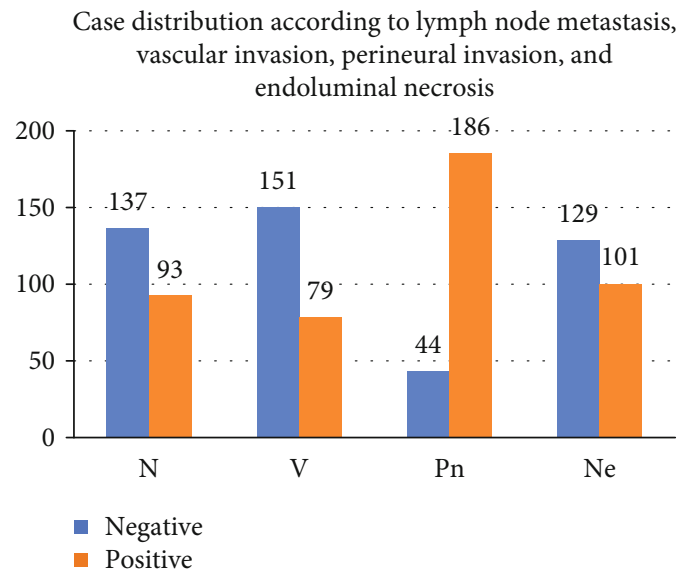

Figure 4: Distribution of lymph node metastasis $(\mathrm{N})$, vascular invasion $(\mathrm{V})$, perineural invasion $(\mathrm{Pn})$, and endoluminal necrosis $(\mathrm{Ne})$.

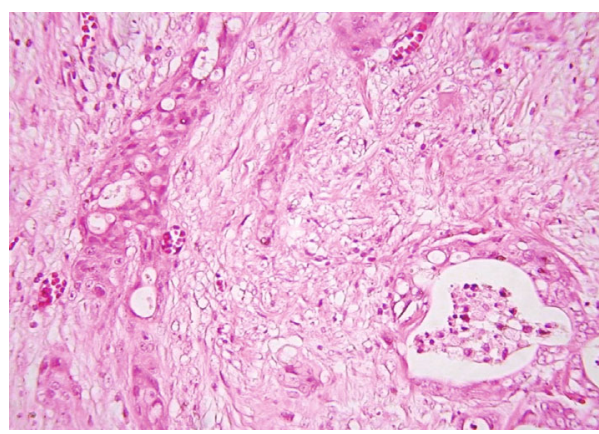

FIgURE 5: Pancreatic ductal adenocarcinoma, endoluminal necrosis, hematoxylin-eosin staining, $\times 200$.

Correlation between pTNM stage and tumor size can be seen in Figure 3.

According to grade distribution, G1 was encountered in $56 \%$ of the cases, G2 was encountered in $29 \%$, and G3 was encountered in $15 \%$.

The other histopathological features observed, namely, the presence/absence of lymph node metastasis (N0/N1), the presence/absence of vascular invasion $(\mathrm{V}+/ \mathrm{V}-)$, the presence/absence of perineural invasion $(\mathrm{Pn}+/ \mathrm{Pn}-)$, and the presence/absence of necrosis in the tumoral glandular lumen $(\mathrm{Ne}+/ \mathrm{Ne})$, are represented in Figures 4 and 5 .

Fifteen out of 230 cases of pancreatic ductal carcinoma were diagnosed as histological variants represented by adenosquamous carcinoma $(n=3)$, mucinous carcinoma $(n=5)$, undifferentiated carcinoma with osteoclast-like giant cells $(n=3)$, and undifferentiated (anaplastic) carcinoma with fusiform cells $(n=4)$.

The positivity of the immunohistochemical markers used is represented in Figure 6 and Table 1.

The microvascular density was appreciated using CD 34 and Factor VIII markers (Figures 7-10). For each case, the same number of serial sections were used, and the maximum distribution of microvascular density, with the cutoff value being 30.1 according literature data [15], was established. All studied features were analyzed depending on two 


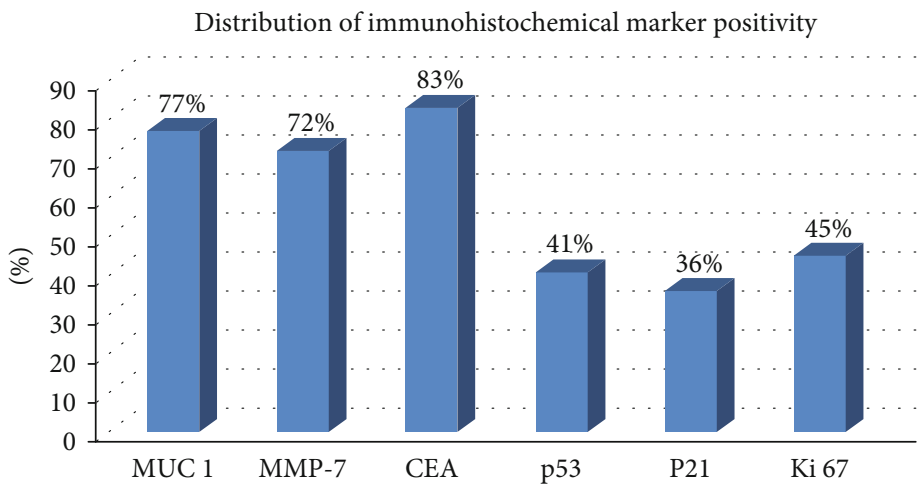

Figure 6: Distribution of immunohistochemical marker positivity.

TABLE 1: Staining index, according to the scale used (0-3+) for the first 3 markers with the highest positivity.

\begin{tabular}{lccc}
\hline & MUC 1 & MMP-7 & CEA \\
\hline 0 & $0-10 \%$ & $0-10 \%$ & $0-15 \%$ \\
$1+$ & $10-35 \%$ & $10-25 \%$ & $15-45 \%$ \\
$2+$ & $35-60 \%$ & $25-50 \%$ & $45-60 \%$ \\
$3+$ & $60-85 \%$ & $50-75 \%$ & $60-85 \%$ \\
\hline
\end{tabular}

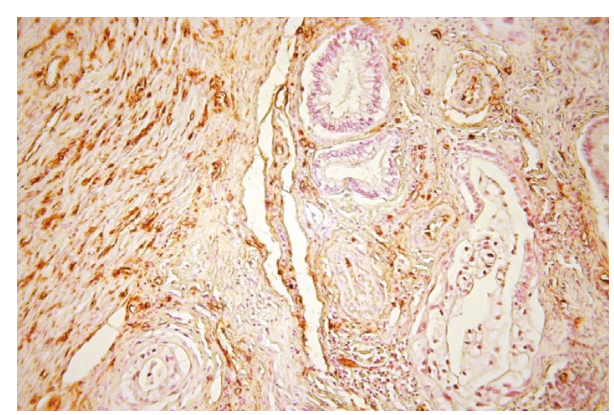

FIgURe 7: Pancreatic ductal adenocarcinoma, hypervascular area, immunohistochemical staining, Factor VIII, $\times 200$.

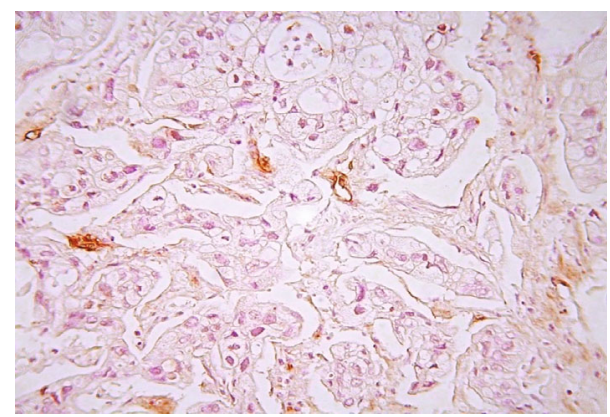

Figure 8: Pancreatic ductal adenocarcinoma, hypovascular area, immunohistochemical staining, Factor VIII, $\times 200$.

categories: hypervascularity and hypovascularity (Table 2). A higher distribution of vessels around the tumor glands was observed (Figures 7 and 9).

$p<0.05$. s.s.: statistically significant; s.i.: statistically insignificant. ${ }^{\mathrm{a}}$ Method used: Student $t$-test. ${ }^{\mathrm{b}}$ Method used: Pearson's chi-squared test.

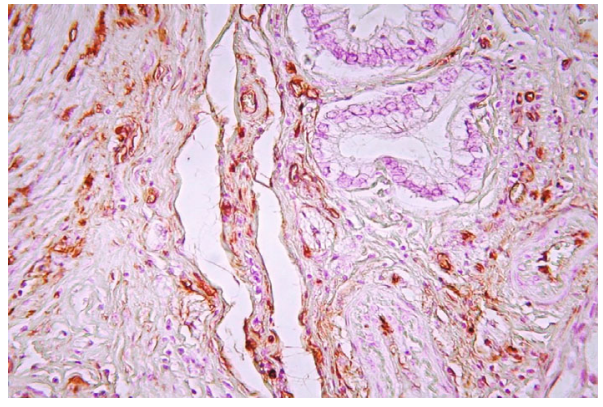

Figure 9: Pancreatic ductal adenocarcinoma, hypervascular area, immunohistochemical staining, CD 34, $\times 200$.

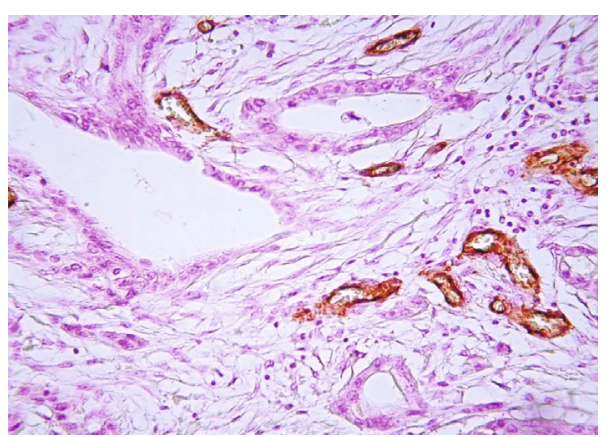

Figure 10: Pancreatic ductal carcinoma, hypovascular area, immunohistochemical staining, CD 34, $\times 200$.

A statistically significant correlation between microvascular density and age, gender, tumor site or size, and presence of tumor metastases could not be established. However, a higher microvascularization in advanced stages was observed (Table 2). For both intratumoral and peritumoral inflammatory infiltrates, we have used an immunohistochemical technique using the antibody for $\mathrm{CD} 68$, which is expressed on macrophages and monocytes.

After data analysis, some of the following correlations could be established (Table 3):

(1) Patients with early stages (IA and IB), with lowgrade malignancy (G1), and with small tumor size $(1-1.5 \mathrm{~cm})$ localized in the head of pancreas; 
TABLE 2: Microvascular analysis according to number of cases and clinicopathological features.

\begin{tabular}{|c|c|c|c|c|}
\hline & \multirow[t]{2}{*}{ No. of cases $(n=230)$} & \multicolumn{2}{|c|}{ Microvascularity } & \multirow[t]{2}{*}{$p$ value* } \\
\hline & & Hypervascularity $(n=108)$ & Hypovascularity $(n=122)$ & \\
\hline Average age (years) & Mean \pm SD & $57.4 \pm 7.9$ & $59.0 \pm 8.3$ & s.i. ${ }^{\mathrm{a}}$ \\
\hline \multicolumn{5}{|l|}{ Gender } \\
\hline $\mathrm{F}$ & $97(42.2 \%)$ & 44 & 53 & s.i ${ }^{b}$ \\
\hline M & $133(57.8 \%)$ & 64 & 69 & s.i. ${ }^{b}$ \\
\hline \multicolumn{5}{|l|}{ Site } \\
\hline Head & $196(85.2)$ & 94 & 102 & s.i. ${ }^{\mathrm{b}}$. \\
\hline Body & $12(5.2 \%)$ & 5 & 7 & s.i. ${ }^{b}$ \\
\hline Tail & $20(8.7 \%)$ & 8 & 12 & s.i. ${ }^{b}$ \\
\hline Panpancreatic & $2(0.9 \%)$ & 1 & 1 & s.i. ${ }^{b}$ \\
\hline \multicolumn{5}{|l|}{ Liver metastasis } \\
\hline Yes & $6(2.6 \%)$ & 4 & 2 & s.i. ${ }^{b}$ \\
\hline No & $224(97.4 \%)$ & 104 & 120 & \\
\hline pT (AJCC) & & & & s.i. ${ }^{b}$ \\
\hline $\mathrm{T} 1$ & $88(38.3 \%)$ & 42 & 46 & \\
\hline $\mathrm{T} 2$ & $127(55.2 \%)$ & 59 & 68 & \\
\hline $\mathrm{T} 3$ & $14(6.1 \%)$ & 6 & 8 & \\
\hline $\mathrm{T} 4$ & $1(0.4 \%)$ & 1 & 0 & \\
\hline Stage & & & & $p=0.005 \mathrm{~s} . \mathrm{s}^{\mathrm{b}}$ \\
\hline IA & $62(27 \%)$ & 24 & 38 & \\
\hline IB & $65(28.3 \%)$ & 22 & 43 & \\
\hline IIA & $9(3.9 \%)$ & 4 & 5 & \\
\hline IIB & $87(37.8 \%)$ & 53 & 34 & \\
\hline III & $1(0.4 \%)$ & 1 & 0 & \\
\hline IV & $6(2.6 \%)$ & 4 & 2 & \\
\hline
\end{tabular}

TAble 3: Prognostic factors according to clinically, histopathologically, and immunohistochemically studied parameters (EN: endoluminal necrosis; ITE: intravascular tumoral emboli).

\begin{tabular}{|c|c|c|c|c|c|c|c|c|c|c|}
\hline Prognosis & Stages & Tumor grade & Tumor size $(\mathrm{cm})$ & Tumor localization & p53 & $\mathrm{p} 21$ & CD 68 & Ki 67 & Metastasis & EN, ITE \\
\hline Better & IA; IB & G1 & $1-1.5$ & Head of pancreas & - & + & $\begin{array}{c}\text { Present in peritumoral } \\
\text { tissue }\end{array}$ & $<5 \%$ & No & No \\
\hline Poor & & G2, G3 & $>5-6$ & $\begin{array}{c}\text { Body or/and tail } \\
\text { Body or/and tail or } \\
\text { head }\end{array}$ & + & - & $\begin{array}{c}\text { Absent in peritumoral } \\
\text { tissue }\end{array}$ & $\begin{array}{l}>30- \\
35 \%\end{array}$ & Yes & Yes \\
\hline
\end{tabular}

p53-negative; p21-positive; CD 68-positive in peritumoral tissue; Ki $67<5 \%$; without metastases at the time of surgery-had a better prognosis with a survival rate of about 7 months. This is why there is a need to focus on improving risk stratification and identifying early stage disease or premalignant lesions, while they are still resectable [7]

(2) Patients with tumors bigger than $5 \mathrm{~cm}$, localized in the body or/and tail; patients with tumors bigger than $5-6 \mathrm{~cm}$, even localized in the head; p21-negative; p53-positive; Ki 67 in 30-35\% of tumoral cells; with endoluminal necrosis, intravascular tumoral emboli, and presence of metastases-had a poor prognosis and increased risk for metastases (when these are absent at the time of diagnosis) and recurrences
(3) Presence of perineural invasion and number of affected regional lymph nodes could not be correlated with prognostic factors or survival [17]

(4) $C D 68$ was positive in peritumoral tissue, in about $55-60 \%$ of well-differentiated G1 cases. The interaction between pancreatic cancer cells and tumorassociated macrophages remains to be established and may play a pivotal role in the progression of pancreatic cancer $[18,19]$

The immunohistochemical panel is useful for an accurate diagnosis, staging, differential diagnosis, and establishment of additional prognostic factors that might be important.

The existence of a viable, stable, and specific marker is mandatory for the detection early disease, for preventing 


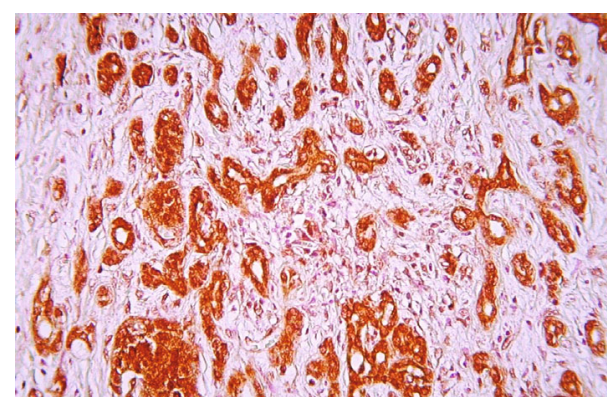

Figure 11: Immunohistochemical staining, MMP-7 (3+), $\times 100$.

recurrences, for prolonging lifetime, and for formulating an adequate therapy.

\section{Discussion}

Pancreatic ductal adenocarcinoma (PDAC) is the most frequent pancreatic neoplasm and represents $80-90 \%$ of all malignant pancreatic tumors [1]. Incidence is about $50 \%$ higher in men than in women and in patients with $60-80$ years of age [1]. In our cases, a slight male predominance has been observed in patients with 50-70 years of age.

According to the literature, the majority of ductal adenocarcinomas are located in the head of pancreas (60-70\%); in our study, the majority of cases $(85.2 \%)$ had the same location $[2-4,8]$.

An important tool in the evaluation of prognosis and guidance of a treatment is pTNM staging $[13,14]$.

The establishment of additional histopathological parameters, besides the usual ones, included in the used classifications, such as the presence of an intravascular tumoral emboli, perineural invasion, and endoluminal necrosis, are useful for the accuracy of the diagnosis $[17,20]$. The presence of numerous intravascular tumoral emboli has to be considered as a poor prognostic factor [21]. In our study, tumoral emboli were present in $59(34.3 \%)$ cases. The presence of lymph node metastases is also an important prognostic factor, without known correlation with the number of affected lymph nodes [16, 22]. In our study, 93(40.4\%) cases had lymph node metastasis. Progression of tumoral stage represents a poor prognostic factor.

We found an increase in microvascularization in advanced stages, and studies have shown that angiogenic markers such as serum levels of VEGF and bFGF correlated with the stage, tumor diameter, and proliferation markers (Ki 67). These markers may also reflect disease progression $[23,24]$.

Most of the studied ductal adenocarcinomas were well differentiated, less than $2 \mathrm{~cm}$, located in the head of the pancreas, without lymph node metastases, and had a better prognosis and a longer survival rate (more than 7 months) Table 3 .

Immunohistochemistry with an increased number of antibodies is very useful; in studied cases, there were antibodies included that underline the following issues:

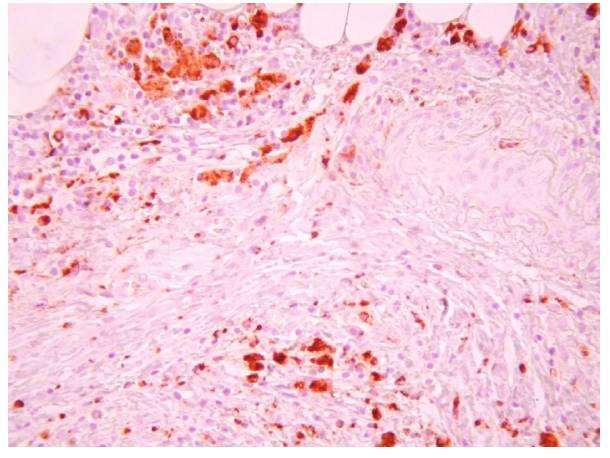

FIgURE 12: Pancreatic ductal carcinoma, inflammatory infiltrate, intratumoral and peritumoral (predominant) tissues, immunohistochemical staining, CD 68, ×200.

(1) MUC 1 was positive in $77 \%$ of the cases, with a staining index between 35 and $85 \%(2+$ and $3+$ on the staining scale) (Table 1), frequently in welldifferentiated cases, independent of the number of lymph node metastases or disease stage. It is expressed usually in the luminal membrane (e.g., in the duct forming areas) and in the cytoplasm (e.g., in poorly differentiated areas); thus, it is considered a marker of aggressiveness [11].

(2) MMP-7 was positive in $72 \%$ of the cases, with a staining index between 25 and $75 \%(2+$ and $3+$ on the staining scale) (Figure 11); it was more frequent in well-differentiated cases, in early stages without lymph node metastases.

(3) $C E A$ was positive in $83 \%$ of the cases, with a staining index between 45 and $85 \%$ ( $2+$ and $3+$ on the staining scale) (Table 1).

(4) p53 was positive in $41 \%$ of the cases (staining index: 1 + and $2+$ ), $25-30 \%$ in stages IA and IB, $45 \%$ in stages IIB and III, and $30-33 \%$ in stage IV; it was negative in adenosquamous carcinoma and mucinous carcinoma (PDC variants). In cases with a 3-5-month survival rate, it was frequently positive; in majority of the cases with a longer survival rate (more than 8 months), it was negative.

(5) P21 was positive in $36 \%$ of the cases, more frequent in early stages and in patients with low-grade carcinoma, G1. P21 is the downstream target of p53 activation and enables the DNA repair mechanisms by inducing G1 arrest. Despite the loss of its activity, its direct role in survival has not been proven yet [25].

(6) $C D 68$ was positive in peritumoral tissue, in about 55 $60 \%$ of the well-differentiated G1 cases (Figure 12).

(7) $\mathrm{Ki} 67$ was positive in about $40-45 \%$ of the cases with high-grade carcinoma (G2, G3) with short survival rate, and in cases with the presence of metastases at the time of surgical resection. There was a correlation between the number of positive cells and tumor's grade and stage. 
A survival rate could be established in only 134 patients (due to lost follow-up, poor communication with oncologists, and lack of response/cooperation from patient's relatives), with an average survival rate of 12.96 months, without a good correlation with studied factors.

In studied cases, PanIN lesions were not as frequently encountered (47\%) as they were presented in the literature, where they might be encountered in until $82 \%$ of cases [26-32]. An explanation for this could be the advanced stage of disease at the time of surgery [31].

\section{Conclusions}

It is recommended to study peritumoral/junction tissue from quantitative and qualitative points of view, to increase the number of prognostic factors.

This study represents a multidisciplinary approach, and it is a result of teamwork. It presents histopathological methods of examination of this severe illness and describes only a part of the scientific effort to determine main pathological mechanisms of this neoplasm.

\section{Data Availability}

All the data to support the findings of this study are available from the corresponding author upon request.

\section{Conflicts of Interest}

The authors declare no conflict of interest.

\section{Acknowledgments}

Part of this research was funded by the project PANCNGS (PN-III-P1-1.2-PCCDI-2017-0797).

\section{References}

[1] F. Bosman, F. Carneiro, R. Hruban, and N. Theise, "WHO Classification of Tumours of the Digestive System (4th edition) Lyon: World Health Organization," International Agency for Research on Cancer, vol. 3, pp. 280-337, 2010.

[2] R. L. Siegel, K. D. Miller, and A. Jemal, "Cancer statistics, 2018," CA: a Cancer Journal for Clinicians, vol. 68, no. 1, pp. 7-30, 2018.

[3] A. Jemal, F. Bray, M. M. Center, J. Ferlay, E. Ward, and D. Forman, "Global cancer statistics," CA: A Cancer J for Clinicians, vol. 61, no. 2, pp. 69-90, 2011.

[4] R. Siegel, J. Ma, Z. Zou, and A. Jemal, "Cancer statistics, 2014," CA : A Cancer Jurnal for Clinicians, vol. 64, no. 1, pp. 9-29, 2014.

[5] M. I. Canto, R. H. Hruban, E. K. Fishman et al., "Frequent detection of pancreatic lesions in asymptomatic high-risk individuals," Gastroenterology, vol. 142, no. 4, pp. 796-804, 2012.

[6] M. Squadroni and N. Fazio, "Chemotherapy in pancreatic adenocarcinoma," European Review for Medical and Pharmacological Sciences, vol. 14, no. 4, pp. 386-394, 2010.

[7] S. A. Badger, J. L. Brant, C. Jones et al., "The role of surgery for pancreatic cancer: a 12-year review of patient outcome," Ulster Medical Jurnal, vol. 79, pp. 70-75, 2010.
[8] D. Michaud, "Epidemiology of pancreatic cancer," Minerva Chirurgica, vol. 59, no. 2, pp. 99-111, 2004.

[9] D. Cao, A. Maitra, J.-A. Saavedra, D. S. Klimstra, N. V. Adsay, and R. H. Hruban, "Expression of novel markers of pancreatic ductal adenocarcinoma in pancreatic nonductal neoplasms: additional evidence of different genetic pathways," Modern Pathology, vol. 18, no. 6, pp. 752-761, 2005.

[10] R. Chen, S. Pan, T. A. Brentnall, and R. Aebersold, "Proteomic profiling of pancreatic cancer for biomarker discovery," Molecular Cell Proteomics, vol. 4, no. 4, pp. 523-533, 2005.

[11] H. C. Harsha, K. Kandasamy, P. Ranganathan et al., "A compendium of potential biomarkers of pancreatic cancer," PLoS Medicine, vol. 6, no. 4, article e1000046, 2009.

[12] C. P. Tanase, M. Neagu, R. Albulescu, E. Codorean, and S. O. Dima, "Biomarkers in the diagnosis and early detection of pancreatic cancer," Expert Opin Med Diagn., vol. 3, no. 5, pp. 533-546, 2009.

[13] UICC, International Union Against Cancer. TNM Classification of Malignant Tumours (seventh ed.), Wiley-Blackwell, 2009.

[14] American Joint Committe on Cancer, Cancer Staging Manual (sixth ed.), Springer, 2002.

[15] K. Fujimoto, R. Hosotani, M. Wada et al., "Expression of two angiogenic factors, vascular endothelial growth factor and platelet-derived endothelial cell growth factor in human pancreatic cancer, and its relationship to angiogenesis," European Jurnal of Cancer, vol. 34, no. 9, pp. 1439-1447, 1998.

[16] J. W. C. Chen, M. Bhandari, D. S. Astill et al., "Predicting patient survival after pancreaticoduodenectomy for malignancy: histopathological criteria based on perineural infiltration and lymphovascular invasion," HPB: The Official Journal of the International Hepato Pancreato Biliary Association, vol. 12, no. 2, pp. 101-108, 2010.

[17] R. H. Hruban, K. Takaori, D. S. Klimstra et al., "An illustrated consensus on the classification of pancreatic intraepithelial neoplasia and intraductal papillary mucinous neoplasms," American Jurnal of Surgical Pathology, vol. 28, no. 8, pp. 977-987, 2004.

[18] F. Meng, C. Li, W. Li, Z. Gao, K. Guo, and S. Song, "Interaction between pancreatic cancer cells and tumor-associated macrophages promotes the invasion of pancreatic cancer cells and the differentiation and migration of macrophages," IUBMB Life, vol. 66, no. 12, pp. 835-846, 2014.

[19] C. Tanase, L. G. Necula, A. I. Neagu, and R. Albulescu, "The association between inflammation and angiogenesis in human pancreatic adenocarcinoma," Journal of Translational Medicine and Research, vol. 21, no. 2, pp. 85-88, 2016.

[20] M. Jhawer, D. Coit, M. Brennan et al., "Perineural invasion after preoperative chemotherapy predicts poor survival in patients with locally advanced gastric cancer: gene expression analysis with pathologic validation," American Journal of Clinical Oncology, vol. 32, no. 4, pp. 356-362, 2009.

[21] S. Yachida, S. Jones, I. Bozic et al., "Distant metastasis occurs late during the genetic evolution of pancreatic cancer," Nature, vol. 467, no. 7319, pp. 1114-1117, 2010.

[22] R. Hruban, "Pancreatic cancer: from genes to patient care," Journal of Gastrointestinal Surgery, vol. 5, no. 6, pp. 583-587, 2001.

[23] C. Pistol-Tanase, E. Raducan, S. O. Dima et al., "Assessment of soluble angiogenic markers in pancreatic cancer," Biomarkers in Medicine, vol. 2, no. 5, pp. 447-455, 2008. 
[24] C. P. Tanase, A. I. Neagu, L. G. Necula et al., "Cancer stem cells: involvement in pancreatic cancer pathogenesis and perspectives on cancer therapeutics," World Journal of Gastroenterology, vol. 20, no. 31, pp. 10790-10801, 2014.

[25] C. P. Tanase, M. Neagu, R. Albulescu, and M. E. Hinescu, "Advances in pancreatic cancer detection," Advances in Clinical Chemistry, vol. 51, pp. 145-180, 2010.

[26] R. H. Hruban, N. V. Adsay, J. Albores-Saavedra et al., "Pancreatic intraepithelial Neoplasia," American Jurnal of Surgical Pathology, vol. 25, no. 5, pp. 579-586, 2001.

[27] E. C. Verna, C. Hwang, P. D. Stevens et al., "Pancreatic cancer screening in a prospective cohort of high-risk patients: a comprehensive strategy of imaging and genetics," Clinical Cancer Research, vol. 16, no. 20, pp. 5028-5037, 2010.

[28] I. Popescu, C. Stroescu, T. Dumitrascu et al., "Digestive tumor bank protocol: from surgical specimens to genomic studies of digestive cancers," Chirurgia, vol. 101, no. 5, pp. 471-475, 2006.

[29] S. O. Dima, C. Tanase, R. Albulescu et al., "An exploratory study of inflammatory cytokines as prognostic biomarkers in patients with ductal pancreatic adenocarcinoma," Pancreas, vol. 41, no. 7, pp. 1001-1007, 2012.

[30] A. Becker, Y. Hernandez, H. Frucht, and A. L. Lucas, "Pancreatic ductal adenocarcinoma: risk factors, screening, and early detection," World Journal of Gastroenterology, vol. 20, no. 32, pp. 11182-11198, 2014.

[31] M. Ionescu, C. Stroescu, S. Ciurea et al., "The value of resection in pancreatic cancer: the analysis of an experience of 180 patients in 10 years," Chirurgia, vol. 99, no. 4, pp. 211-220, 2004.

[32] M. Goggins, "Molecular markers of early pancreatic cancer," Jurnal of Clinical Oncology, vol. 23, no. 20, pp. 4524-4531, 2005. 